\title{
High expression of MMP9 in glioma affects cell proliferation and is associated with patient survival rates
}

\author{
QIANG XUE ${ }^{1 *}$, LI CAO $^{2 *}$, XIAO-YAN CHEN $^{3 *}$, JING ZHAO $^{4 *}$, LIANG GAO $^{5}$, SAN-ZHONG LI $^{3}$ and ZHOU FEI ${ }^{3}$ \\ Departments of ${ }^{1}$ Cardiology, ${ }^{2}$ Human Resources, ${ }^{3}$ Neurosurgery, ${ }^{4}$ Anesthesiology and ${ }^{5}$ Ultrasound, \\ Xijing Hospital, Fourth Military Medical University, Xi'an, Shaanxi 710032, P.R. China
}

Received July 16, 2015; Accepted November 10, 2016

DOI: $10.3892 / \mathrm{ol} .2017 .5567$

\begin{abstract}
Human gliomas are a heterogeneous group of primary malignant brain tumors, which most commonly occur in the central nervous system of children and adults. Previous studies have suggested a prognostic role of matrix metalloproteinase 9 (MMP9) in glioma, however, the frequency and significance of the protein expression of MMP9 in glioma remain to be fully elucidated. In the present study, the expression of MMP9 was detected by reverse transcription-quantitative polymerase chain reaction (qPCR), western blotting and immunohistochemical staining. MTT and colony-forming assays were used to detect the role of MMP9 in the proliferation of glioma cells. MMP9 copy numbers in glioma were examined using qPCR. The results indicated that the expression level of MMP9 was significantly increased in glioma and was associated with World Health Organization (WHO) glioma grades. The high expression of MMP9 in tissues was an independent predictor of survival rates in patients with WHO grade III tumors. The overexpression of MMP9 promoted cell growth and induced a significant increase in clonogenic potential in U87 glioblastoma cell lines. These experimental data suggested that the overexpression of MMP9 in glioblastoma cells may occur primarily through an increase in gene copy number. The results of the present study
\end{abstract}

Correspondence to: Dr San-Zhong Li or Dr Zhou Fei, Department of Neurosurgery, Xijing Hospital, Fourth Military Medical University, 169 Changle West Road, Xi'an, Shaanxi 710032, P.R. China

E-mail: sanzhong_li@163.com

E-mail: feizhou@fmmu.edu.cn

*Contributed equally

Abbreviations: MMP9, matrix metalloproteinase 9; RT-qPCR, reverse transcription-quantitative polymerase chain reaction; WHO, World Health Organization; GBM, glioblastoma multiforme; ECM, extracellular matrix; FBS, fetal bovine serum; RNA, ribonucleic acid; cDNA, complementary DNA

Key words: matrix metalloproteinase 9, glioma, prognosis, cell proliferation suggested that the overexpression of MMP9 may be necessary for the transition to the more aggressive phenotype typical of WHO grade III gliomas, suggesting the likely involvement of the MMP9 gene in gliomagenesis and disease progression.

\section{Introduction}

Human gliomas are a heterogeneous group of primary malignant brain tumors, which most commonly occur in the central nervous system of children and adults (1). Glioblastoma multiforme (GBM), the most aggressive form of glioma, exhibits advanced features of malignancy, including rapid tumor cell proliferation, apoptosis resistance, florid necrosis and angiogenesis (2). These tumor properties are associated with poor clinical outcome by conferring resistance to chemotherapy and radiotherapy, and by promoting neurological debilitation leading to individuals succumbing to mortality within 12-18 months of diagnosis (3).

Gliomas can be categorized based on the type of glial cell, which they are most histologically similar to, the location of the tumor and the aggressiveness of the cancer cells. Tumors, which are most similar to astrocytes are specifically termed astrocytomas and can be further classified into grades I-IV based on the criteria set by the World Health Organization (WHO) (4), with higher grades corresponding to more aggressive tumors. Grade I and II astrocytomas correspond to low-grade tumors, which are predominantly non-malignant. Grade III and IV astrocytomas are high-grade, malignant tumors. Grade III astrocytomas are also known as anaplastic astrocytomas, whereas grade IV astrocytomas, commonly referred to as glioblastoma, are the most aggressive of all gliomas. GBMs are also the most common type of glioma with an annual incident rate of 3.19/100,000 in the USA $(5,6)$.

Matrix metalloproteinases (MMPs) are zinc-dependent endopeptidases, and their expression is regulated by proteolytic activation and by selective inhibitory proteins. The majority of the extracellular matrix (ECM) components are substrates of MMPs (7). MMPs have also been reported to process several bioactive factors, apoptotic chemokines and cell signaling factors, which affect immune responses (8). The overexpression of certain members of the MMP family has been correlated with invasion, metastasis and poor prognosis. MMP9 has been implicated in the invasion and metastasis of head and neck squamous cell carcinoma $(9,10)$. The knockout 
of MMP9 has been reported to lead to reduced skin and pancreatic carcinogenesis and metastasis with delayed tumor vascularization $(10,11)$. In addition, the knockout of MMP 23, 28 and 29 lead to enhanced tumorigenesis and metastasis in certain animal models of cancer (11). The present study hypothesized that MMP9 may be an important factor in the progression and prognosis of glioma through proliferation and angiogenesis.

The aim of the present study was to investigate the role of MMP9 in glioma. The results demonstrated that MMP9 was upregulated in glioma tissues and its expression was correlated with tumor grade. It was also observed that the overexpression of MMP9 in a glioma cell line accelerated tumor growth and induced a significant increase in clonogenic potential. It was shown that an increase in the number of copies of MMP was significantly associated with the increased expression of MMP in gliomas.

\section{Materials and methods}

Tissue specimens and patients. Tumor tissues were collected from 62 patients with gliomas who underwent successful tumor resection or biopsy at the Department of Neurosurgery of Xijing Hospital, Fourth Military Medical University (Xi'an, China) between March 2012 and March 2015. The patients included 45 men and 17 women, with a median age of 62 years (range, 32-88 years). In addition, the results of survival analyses were collected from 80 patients with grade III gliomas who underwent successful tumor resection or biopsy at the Department of Neurosurgery of Xijing Hospital, Fourth Military Medical University between July 2001 and July 2005. The present study was performed in accordance with The Code of Ethics of the World Medical Association (Declaration of Helsinki) and approved by the Institutional Review Board of Xijing Hospital, Fourth Military Medical University. Written informed consent was obtained from each patient or their legally authorized representative. All patients underwent preoperative computed tomography and magnetic resonance imaging. All tissue sections were reviewed by two pathologists without knowledge of clinical outcomes. Following collection during surgery, half of the tissue sample from the bulk of the tumor was immediately frozen in liquid nitrogen and stored at $-80^{\circ} \mathrm{C}$. The remaining half was processed for primary tumor cultures.

Cell culture and transfection. The human U87 glioma cells (American Type Culture Collection, Manassas, VA, USA) were maintained in RPMI 1640 medium supplemented with $10 \%$ fetal bovine serum (FBS; Sijiqing, Hangzhou, China), $2 \mathrm{mM}$ L-glutamine, $50 \mathrm{IU} / \mathrm{ml}$ penicillin and $50 \mu \mathrm{g} / \mathrm{ml}$ streptomycin sulfate, and cultured in $5 \% \mathrm{CO}_{2}$ at $37^{\circ} \mathrm{C}$. The U87 cells were transfected with $2 \mu \mathrm{g}$ of pIRES2-enhanced green fluorescent protein (EGFP)-MMP9 (Clontech Laboratories, Inc., Mountainview, CA, USA) or pIRES2-EGFP (Clontech Laboratories, Inc.) using $5 \mu$ l Lipofectamine 2000 reagent (Invitrogen; Thermo Fisher Scientific, Inc.) according to the manufacturer's protocol. At $5 \mathrm{~h}$ post-transfection, the medium was replaced with complete medium, and the cells were incubated for a further $48 \mathrm{~h}$. The cells were then selected by G418 (1 $\mathrm{mg} / \mathrm{ml}$ concentration). After 2 weeks, the G418-resistant colonies were isolated and pooled, and were confirmed using fluorescence-activated cell sorting, showing that $>90 \%$ cells were EGFP positive.

MTT and colony-forming assays. The U87 cells were seeded in triplicate in 96-well plates $\left(1 \times 10^{3}\right.$ cells/well) and were cultured in $200 \mu \mathrm{l}$ medium for $48 \mathrm{~h}$ prior to the addition of MTT. For the MTT assay, half of the medium (100 $\mu \mathrm{l})$ was removed and an equal volume of fresh medium containing $20 \%$ MTT $(5 \mathrm{mg} / \mathrm{ml})$ was added. The cells were incubated for a further $4 \mathrm{~h}$ at $37^{\circ} \mathrm{C}$. The medium was then removed, and $150 \mu 1$ of dimethyl sulfoxide (Sigma-Aldrich; Merck Millipore, Darmstadt, Germany) was added to each well, and mixed by shaking at room temperature for $10 \mathrm{~min}$. The absorbance was measured at $490 \mathrm{~nm}$. To perform a colony-forming assay, the U87 cells $\left(2 \times 10^{3} /\right.$ well $)$ were embedded in medium containing $0.33 \%$ agar gel and $10 \%$ FBS in 12-well plates, pre-coated with $0.5 \%$ agar solution, in triplicate. The gel was covered with normal medium and was cultured routinely. The number of colonies, defined as containing $>50$ cells, were counted following 10 days of incubation under an inverted microscope (IX71; Olympus Corporation, Tokyo, Japan).

Reverse transcription-quantitative polymerase chain reaction (RT-qPCR) analysis. Total RNA was extracted from the cultured cells using TRIzol reagent (Invitrogen; Thermo Fisher Scientific, Inc.) according to the manufacturer's protocol. Complementary DNA (cDNA) was prepared using a reverse transcription system (Toboyo Co., Ltd., Osaka, Japan) according to the manufacturer's protocol. qPCR was performed in triplicate using a SYBR Premix EX Taq kit (Takara Bio, Inc., Otsu, Japan) and the ABI PRISM 7300 real-time PCR system (Applied Biosystems; Thermo Fisher Scientific, Inc.) with GAPDH as an internal control. The total reaction system consisted of $30 \mu \mathrm{l}$ with $15 \mu \mathrm{l}$ SYBR Premix, $1 \mu \mathrm{l}$ cDNA, $1 \mu \mathrm{l}$ upstream primer, $1 \mu \mathrm{l}$ downstream primer and $12 \mu \mathrm{l}$ double-distilled $\mathrm{H}_{2} \mathrm{O}$. The thermocycling conditions were as follows: $95^{\circ} \mathrm{C}$ for $1 \mathrm{~min}$, followed by $95^{\circ} \mathrm{C} 30 \mathrm{sec}$ and $60^{\circ} \mathrm{C}$ for $34 \mathrm{sec}$ for 35 cycles. The following primers for were used for qPCR: GAPDH, forward 5'-GCACCGTCAAGG CTGAGAAC-3' and reverse 5'-TGGTGAAGACGCCAG TGGA-3'; and MMP9, forward 5'-GTGCTGGGCTGCTGC TTTGCTG-3' and reverse 5'-GTCGCCCTCAAAGGTTTG GAAT-3'. RT-qPCR quantification was performed with the $2^{-\Delta \Delta \mathrm{Cq}}$ method (12).

Analysis of copy number variation. Genomic DNA was extracted from the fresh and frozen glioma tissues using an automated DNA extractor (EZ1; Qiagen GmbH, Hilden, Germany) and quantified using a Nanodrop spectrophotometer (Thermo Fisher Scientific, Inc.). Sequencing of the MMP9 coding region was performed in 62 patients. Primers were designed using Primer 5.0 software to amplify the five coding exons of the MMP9 (RefSeq, https://www.ncbi.nlm.nih. gov/gene/4318; NM_004994.2) gene, including the intronic flanking sequences. The primer sequences were as follows: MMP9 forward, 5'-TACTCTGCCTGCACCACCGA-3' and reverse 5'-TCTCTCATCATTTCTCAGAT-3'. The amplified products were subsequently purified and sequenced. For the analysis of variations in MMP9 gene copy number, four 
normalization assays mapping to HSA21 and four normalization DNAs were systematically included in each run, as described previously (13). Gene dosage segments were classified as chromosomal 'gain' or 'loss' if the absolute value of the predicted dosage was $>0.75$ times the interquartile range of the difference between the observed and predicted values for each region. The sequencing reactions were performed as reported previously (14).

Immunohistochemistry. The antibodies used for immunohistochemistical analysis included rabbit polyclonal anti-MMP9 antibody (cat. no. ab38898; Abcam, Cambridge, MA, USA) and peroxidase goat anti-rabbit IgG antibody (cat. no. PI-1000; Vector Laboratories, Inc., Burlington, CA, USA). Immunohistochemical staining was performed using peroxidase complex methods. Tissue sections ( $4 \mu \mathrm{m}$ thick) were mounted on slides, and then deparaffinized and rehydrated through xylene baths and graded concentrations of alcohol. Antigen retrieval was performed via pressure cooker treatment at $100^{\circ} \mathrm{C}$ for $100 \mathrm{sec}$ in $0.01 \mathrm{mmol} / 1$ citrate buffer $(\mathrm{pH} 6)$. The sections were immersed in $0.3 \%$ hydrogen peroxide for $12 \mathrm{~min}$ to inactivate the endogenous peroxidase, and then incubated with $10 \%$ blocking serum for $30 \mathrm{~min}$ to reduce nonspecific binding. The primary antibody against MMP9 was diluted at 1:400, and the sections were incubated with the diluted primary antibody overnight at $4^{\circ} \mathrm{C}$. Subsequently, the slides were incubated with the secondary antibody (peroxidase goat anti-rabbit IgG antibody, diluted 1:2,000) at room temperature for $2 \mathrm{~h}$. Diaminobenzidine was used as a chromogen, and commercial hematoxylin was used for counterstaining. The staining was visualized with a BX51 microscope (Olympus Corporation).

Western blot analysis. The glioma tissues were minced in RIPA lysis buffer (Beyotime Institute of Biotechnology, Inc., Haimen, China) containing 0.1 mM PMSF. The tissue extracts were collected by centrifugation at $14,000 \times \mathrm{g}$ for $5 \mathrm{~min}$ at $4^{\circ} \mathrm{C}$. The protein concentration in the extracts was determined using BCA Protein Assay reagents (Pierce; Thermo Fisher Scientific, Inc.), according to the manufacturer's protocol. The samples (20 $\mu \mathrm{g} /$ lane) were analyzed using $12 \%$ SDS polyacrylamide gel electrophoresis, followed by blotting onto a nitrocellulose membrane. The membranes were probed using primary antibodies, followed by horseradish peroxidase-conjugated goat anti-mouse or rabbit antibodies. The primary antibodies included rabbit polyclonal IgG anti-MMP9 (cat. no. ab38898; Abcam) and monoclonal anti- $\beta$-actin (cat. no. ABT264; Sigma-Aldrich; Merck Millipore). The primary antibodies were diluted 1:1,000 and incubated overnight at $4^{\circ} \mathrm{C}$. The secondary antibody (peroxidase goat anti-rabbit IgG antibody; cat. no. PI-1000; Vector Laboratories, Inc.) was diluted 1:4,000 and incubated for $2 \mathrm{~h}$ at room temperature. The membrane was developed using chemoluminescent reagents (SuperSingnal West Femto Maximum Sensitivity Substrate; Pierce; Thermo Fisher Scientific, Inc.) and quantification was performed using Tanon 5200 Multi software (Tanon Science \& Technology Co., Ltd. Shanghai, China).

Statistical analysis. SPSS 13.0 software (SPSS, Inc., Chicago, IL, USA) was used for statistical analysis. The results are expressed as the mean \pm standard deviation. Differences in
A

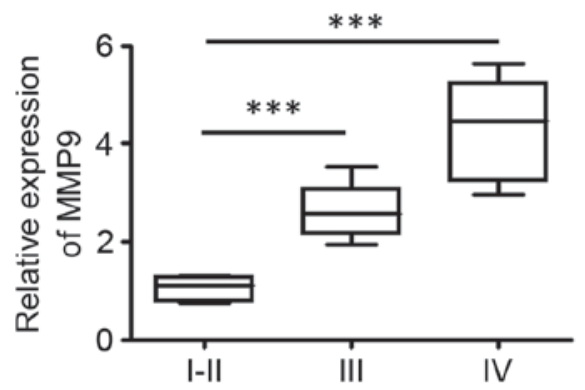

B

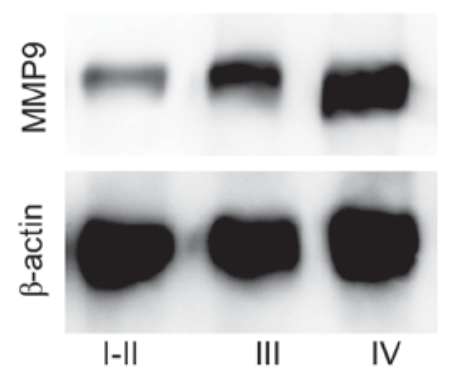

C

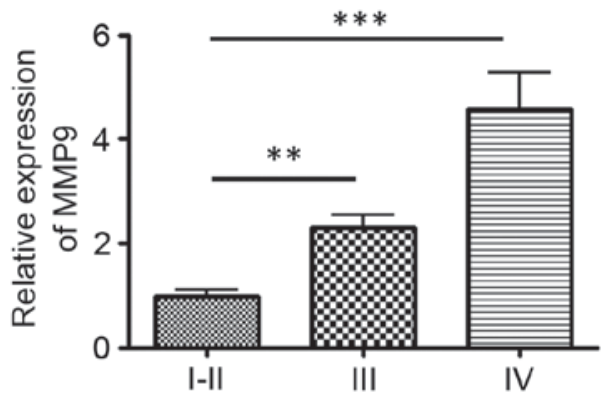

Figure 1. MMP9 is upregulated in higher-grade gliomas. (A) Box-plots of tissue expression levels of MMP9 between tumor grades. Reverse transcription-quantitative polymerase chain reaction analysis was performed to measure the mRNA levels of MMP9 in a total of 62 glioma tissues. (B) Protein expression levels of MMP9 in glioma tissues of each tumor grade were detected using western blot analysis and (C) compared ( ${ }^{* *} \mathrm{P}<0.01$ and $\left.{ }^{* * * *} \mathrm{P}<0.001\right)$. MMP9, matrix metalloproteinase 9 .

the expression of MMP9 in glioma tissues between tumor grades (WHO grades II, III and IV) were assessed using analysis of variance. The difference in survival rates between the two groups, shown by Kaplan-Meier curves, was examined using a log-rank test. Student's $t$-test was used to determine the difference in absorbance and number of colonies between two groups. Histograms of MMP9 copy number frequency distributions with respect to tumor grades were produced. The linear trend of MMP9 copy numbers across tumor grades was determined using the Mantel-Haenszel $\chi^{2}$-test. $\mathrm{P}<0.05$ was considered to indicate a statistically significant difference.

\section{Results}

MMP9 is upregulated in higher-grade gliomas. The RNA expression level of MMP9 was determined from 62 glioma tissues, comprising $11 \mathrm{WHO}$ grades I and II, $18 \mathrm{WHO}$ grade III and 33 WHO grade IV. The relative expression levels 
$\mathbf{A}$

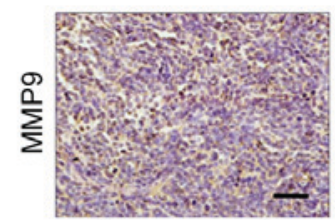

Low expression

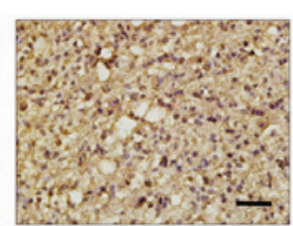

High expression

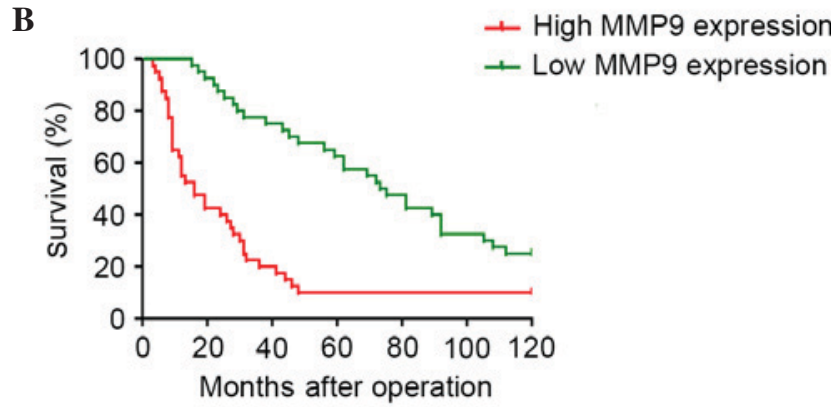

Figure 2. High tissue expression levels of MMP9 in World Health Organization grade III gliomas are associated with poor survival rates. (A) Immunohistochemical staining for MMP9 in human glioma tissues. Images show low expression (20\%) and high expression of MMP9. Magnification, x200. (B) Kaplan-Meier curves for overall survival rates according to the expression levels of MMP9 in patients with grade III tumors. MMP9, matrix metalloproteinase 9 .

of MMP9 ranged between 0.75 and 5.62 in the tumor tissues. A statistically significant higher relative expression of MMP9 was found in grade IV tumor tissues, compared with grades I-II and III $(\mathrm{P}<0.001)$ tumor tissues (Fig. 1A). In the glioma tumor tissues, the median relative expression of MMP9 was 4.28 in GBM with an interquartile range (IQR) of 2.96-5.62, 2.61 in WHO grade III (IQR, 1.95-3.52) and 0.85 in WHO grade I-II (IQR, 0.55-1.61) glioma (Fig. 1A). The protein expression levels of MMP9 in these glioma tissues were then examined. There was also a statistically significant higher relative expression in grade IV tumor tissues, compared with grades I-II and III tumor tissues $(\mathrm{P}<0.001$; Fig. $1 \mathrm{~B}$ and $\mathrm{C})$. These results indicated that MMP9 was upregulated in gliomas of higher grade.

High tissues expression levels of MMP9 are associated with unfavorable clinical outcomes in paitnets with WHO grade III gliomas. The present study detected the expression of MMP9 in glioma tumor samples. The median expression level of MMP9 was 50\% (mean, 50\%; range, 10-80\%; Fig. 2A). The association between the expression of MMP9 and overall survival rate was examined in 80 patients with grade III gliomas, who underwent successful tumor resection or biopsy at the Department of Neurosurgery of Xijing Hospital, Fourth Military Medical University between March 2001 and March 2005. Univariate analysis was performed using a log-rank test, which indicated that patients with a high expression of MMP9 had a significantly lower overall survival rate, compared with those with a low expression of MMP9 (Fig. 2B). These data suggested that the expression levels of MMP9 may represent an independent predictor of survival rates in patients with WHO grade III gliomas.

MMP9 induces cell proliferation in glioblastoma. To evaluate the effect of MMP9 in glioma cell proliferation, U87

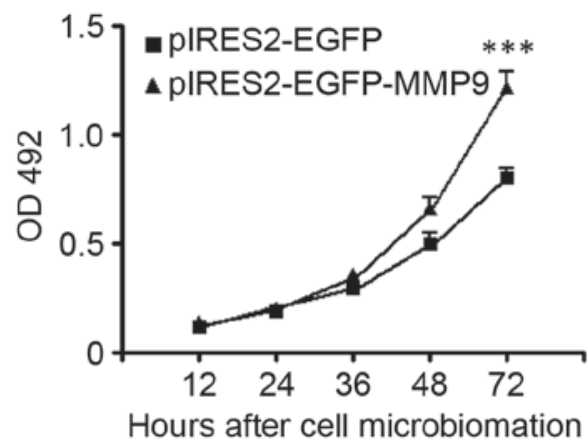

B

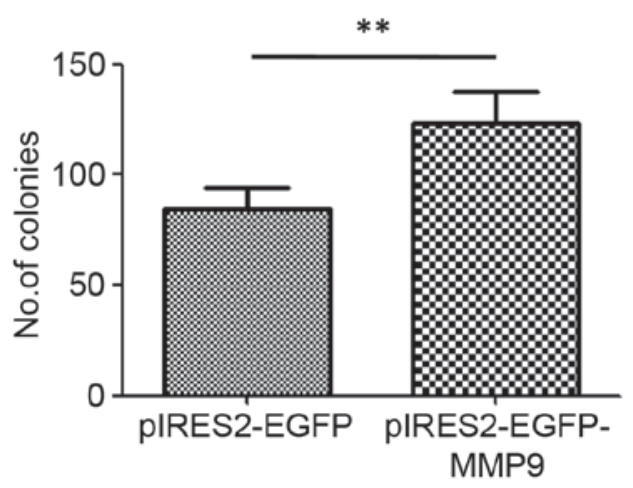

Figure 3. MMP9 induces cell proliferation in glioblastomas. (A) U87 glioblastoma cell lines were stably transfected with pIRES2-EGFP-MMP9 or pIRES2-EGFP. Cells were cultured and cell proliferation was assessed using a methylthiazolyldiphenyl tetrazolium assay. (B) Colony forming assay. Cells were cultured in soft agar, and the formation of colonies was observed and compared $\left({ }^{* *} \mathrm{P}<0.01\right.$ and $\left.{ }^{* * * *} \mathrm{P}<0.001 ; \mathrm{n}=5\right)$. MMP9, matrix metalloproteinase 9; OD, optical density; EGFP, enhanced green fluorescent protein.

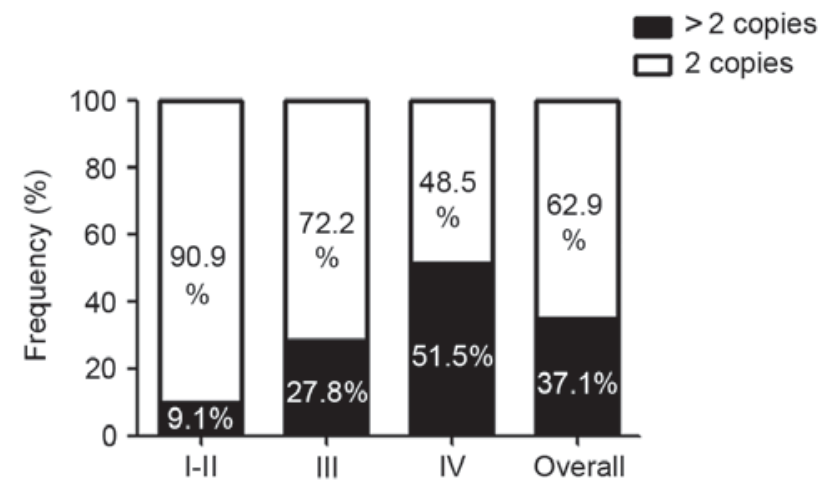

Figure 4. MMP9 copy number is increased in glioma. The MMP9 copy numbers in the gliomas were examined using quantitative polymerase chain reaction analysis on DNA extracts from patient glioma tissues. The frequency distribution of MMP9 copy number is shown within each tumor grade. MMP9, matrix metalloproteinase 9.

glioma cells were transfected with pIRES2-EGFP-MMP9 to construct a cell line stably expressing MMP9. The cell viability was measured using an MTT assay at 12, 24, 36, 48 and $72 \mathrm{~h}$ post-microbiomation, respectively. The results showed that the overexpression of MMP9 significantly increased cell proliferation $(\mathrm{P}<0.001$; Fig. 3A). Subsequently, the present investigated whether the overexpression of MMP9 affected the clonogenic potential of glioma cells as an indirect 
index of their tumorigenic potential. The U87 glioma cells, transfected to express MMP9 or with a control vector, were plated at limiting dilution and the formation of large colonies (>50 cells) was assessed 10 days later. The enforced expression of MMP9 induced a significant increase in the clonogenic potential $(\mathrm{P}<0.01)$, with an average of 123.3 colonies in the MMP9-transfected cells, compared with 84.7 colonies in the control-transfected cultures (Fig. 3B).

Increase in MMP9 copy numbers in glioma. To investigate the mechanisms, which may cause the increase in the expression of MMP9 in glioma, the coding and untranslated regions of MMP9 were sequenced in 62 patients, detecting any likely pathogenic variant (data not shown). Subsequently, to determine MMP9 copy numbers, RT-qPCR analysis was performed. The MMP9 gene copy number increased in 23/62 (37.1\%) of the analyzed glioma tissue samples. A linear trend of MMP9 copy number across tumor grades was found (Fig. 4). Specifically, an increase in MMP9 copy number was observed in $\sim 9.1 \%$ of the grade I and II gliomas, $27.8 \%$ of the grade III gliomas of and $51.5 \%$ of the glioblastomas (Fig. 4).

\section{Discussion}

MMPs are critical in tumor cell growth, migration, invasion, metastasis and angiogenesis (15). MMP9 functions predominantly as a collagenase by degrading type IV collagen, a major component of the ECM and basement membrane $(16,17)$. In view of its broad functions in tumor invasion and metastasis, MMP9 may be a valuable prognostic biomarker in glioma. Previous meta-analysis has shown a significant correlation between high expression levels of MMP9 and poor prognosis in gastric cancer (18), breast cancer (19), non-small cell lung cancer (20) and colorectal cancer (21). MMP9 has also been implicated in the invasion and metastasis of head and neck squamous cell carcinoma (9). Knockout of MMP9 leads to reduced skin and pancreatic carcinogenesis and metastasis, showing delayed tumor vascularization (9-11). In the present study, the prognostic value of MMP9 in glioma was investigated. It was hypothesized that MMP9 may be a valuable factor in determining the progression and prognosis of gliomas.

Human gliomas are the most common and life-threatening type of neurological malignancy in adults (22). The present study was the first, to the best of our knowledge, to investigate the role of MMP9 in glioma. The experimental evidence showed that the expression levels of MMP9 were significantly increased in glioma and were associated with glioma WHO grades in the tissues collected. A higher expression levels of MMP9 in tissues was an independent predictor of survival rates in WHO grade III tumors. In addition, the overexpression of MMP9 promoted cell growth and induced a significant increase in the clonogenic potential of U87 glioblastoma cell lines. The present study also investigated the molecular mechanism underlying the observed increase in the expression of MMP9. The experimental data suggested that the overexpression of MMP9 in glioblastoma cells may have occurred primarily through the increase in gene copy number. In the present study, the analysis of survival rates revealed a significant increase in disease progression and decrease in survival rates of patients with WHO grade III tumors expressing higher levels of MMP9, compared with those with lower expression levels. These results suggested that the overexpression of MMP9 may be necessary for the transition to the aggressive phenotype typical of WHO grade III gliomas, suggesting the likely involvement of the MMP9 gene in gliomagenesis and disease progression.

\section{Acknowledgements}

This study was supported by the Social Development Research project in Shaanxi province, China (grant no. 2010K14-02-05). The authors would like to thank the members of the laboratories at the Department of Neurosurgery, Xijing Hospital of the Fourth Military Medical University.

\section{References}

1. Marumoto T and Saya H: Molecular biology of glioma. Adv Exp Med Biol 746: 2-11, 2012.

2. Ohgaki H, Dessen P, Jourde B, Horstmann S, Nishikawa T, Di Patre PL, Burkhard C, Schüler D, Probst-Hensch NM, Maiorka PC, et al: Genetic pathways to glioblastoma: A population-based study. Cancer Res 64: 6892-6899, 2004.

3. Furnari FB, Fenton T, Bachoo RM, Mukasa A, Stommel JM, Stegh A, Hahn WC, Ligon KL, Louis DN, Brennan C, et al: Malignant astrocytic glioma: Genetics, biology, and paths to treatment. Genes Dev 21: 2683-2710, 2007.

4. Hervey-Jumper SL and Berger MS: Maximizing safe resection of low- and high-grade glioma. J Neurooncol 130: 269-282, 2016.

5. Ostrom QT, Gittleman H, Liao P, Rouse C, Chen Y, Dowling J, Wolinsky Y, Kruchko C and Barnholtz-Sloan J: CBTRUS statistical report: Primary brain and central nervous system tumors diagnosed in the United States in 2007-2011. Neuro Oncol 16 (Suppl 4): iv1-iv63, 2014.

6. Thomas AA, Brennan CW, DeAngelis LM and Omuro AM: Emerging therapies for glioblastoma. JAMA Neurol 71: 1437-1444, 2014

7. Husmann K, Arlt MJ, Muff R, Langsam B, Bertz J, Born W and Fuchs B: Matrix Metalloproteinase 1 promotes tumor formation and lung metastasis in an intratibial injection osteosarcoma mouse model. Biochim Biophys Acta 1832: 347-354, 2013.

8. Korpi JT, Hagström J, Lehtonen N, Parkkinen J, Sorsa T, Salo T and Laitinen M: Expression of matrix metalloproteinases-2, -8, $-13,-26$, and tissue inhibitors of metalloproteinase- 1 in human osteosarcoma. Surg Oncol 20: e18-e22, 2011.

9. Deraz EM, Kudo Y, Yoshida M, Obayashi M, Tsunematsu T, Tani H, Siriwardena SB, Keikhaee MR, Qi G, Iizuka S, et al: MMP-10/stromelysin-2 promotes invasion of head and neck cancer. PLoS One 6: e25438, 2011.

10. Folgueras AR, Pendás AM, Sánchez LM and López-Otin C: Matrix metalloproteinases in cancer: From new functions to improved inhibition strategies. Int J Dev Biol 48: 411-424, 2004.

11. Overall CM and Kleifeld O: Tumour microenvironment-opinion: Validating matrix metalloproteinases as drug targets and anti-targets for cancer therapy. Nat Rev Cancer 6: 227-239, 2006.

12. Livak KJ and Schmittgen TD: Analysis of relative gene expression data using real-time quantitative PCR and the 2(-Delta Delta C(T)) Method. Methods 25: 402-408, 2001.

13. Howald C, Merla G, Digilio MC, Amenta S, Lyle R, Deutsch S, Choudhury U, Bottani A, Antonarakis SE, Fryssira H, et al: Two high throughput technologies to detect segmental aneuploidies identify new Williams-Beuren syndrome patients with atypical deletions. J Med Genet 43: 266-273, 2006.

14. Micale L, Augello B, Fusco C, Selicorni A, Loviglio MN, Silengo MC, Reymond A, Gumiero B, Zucchetti F, D'Addetta EV, et al: Mutation spectrum of MLL2 in a cohort of Kabuki syndrome patients. Orphanet J Rare Dis 6: 38, 2011.

15. Egeblad M and Werb Z: New functions for the matrix metalloproteinases in cancer progression. Nat Rev Cancer 2: 161-174, 2002.

16. Liotta LA, Tryggvason K, Garbisa S, Hart I, Foltz CM and Shafie S: Metastatic potential correlates with enzymatic degradation of basement membrane collagen. Nature 284: 67-68, 1980.

17. Stetler-Stevenson WG: Type IV collagenases in tumor invasion and metastasis. Cancer Metastasis Rev 9: 289-303, 1990. 
18. Zhang QW, Liu L, Chen R, Wei YQ, Li P, Shi HS and Zhao YW: Matrix metalloproteinase- 9 as a prognostic factor in gastric cancer: A meta-analysis. Asian Pac J Cancer Prev 13: 2903-2908, 2012.

19. Song J, Su H, Zhou YY and Guo LL: Prognostic value of matrix metalloproteinase 9 expression in breast cancer patients: A meta-analysis. Asian Pac J Cancer Prev 14: 1615-1621, 2013.

20. Peng WJ, Zhang JQ, Wang BX, Pan HF, Lu MM and Wang J: Prognostic value of matrix metalloproteinase 9 expression in patients with non-small cell lung cancer. Clin Chim Acta 413: 1121-1126, 2012.
21. Li CY, Yuan P, Lin SS, Song CF, Guan WY, Yuan L, Lai RB, Gao Y and Wang Y: Matrix metalloproteinase 9 expression and prognosis in colorectal cancer: A meta-analysis. Tumour Biol 34: 735-741, 2013.

22. Micale L, Fusco C, Fontana A, Barbano R, Augello B, De Nittis P, Copetti M, Pellico MT, Mandriani B, Cocciadiferro $\mathrm{D}$, et al: TRIM8 downregulation in glioma affects cell proliferation and it is associated with patients survival. BMC Cancer 15: 470, 2015. 